\title{
Prognostic impact of venous invasion in stage IB node-negative gastric cancer
}

\author{
Ippeita Araki $\cdot$ Kei Hosoda $\cdot$ Keishi Yamashita $\cdot$ Natsuya Katada $\cdot$ \\ Shinichi Sakuramoto · Hiromitsu Moriya · Hiroaki Mieno · Akira Ema • \\ Shiro Kikuchi · Tetuo Mikami • Masahiko Watanabe
}

Received: 9 December 2013/Accepted: 23 February 2014/Published online: 1 April 2014

(c) The International Gastric Cancer Association and The Japanese Gastric Cancer Association 2014

\begin{abstract}
Background Little is known about risk factors for recurrence in stage IB gastric cancer without lymph node metastasis. The aims of this study were to determine prognostic factors associated with long-term survival and to clarify patterns of recurrence.

Methods We retrospectively reviewed the medical records of 130 patients with primary gastric cancer who underwent gastrectomy at Kitasato University East Hospital from 2001 through 2010 and analyzed clinicopathological characteristics associated with survival and patterns of recurrence.

Results Of the 130 patients, $12(9.2 \%)$ had recurrence, among whom $10(83 \%)$ patients died. Four patients (3.1\%) died of other diseases. The 5-year overall survival rate was $89 \%$. Of the 12 patients with recurrence, 7 (58 \%) had liver metastasis, $3(25 \%)$ had distant lymph-node metastasis, $2(17 \%)$ had peritoneal dissemination, and 1 $(8.3 \%)$ had locoregional recurrence. Patients with tumors more than $5 \mathrm{~cm}$ in diameter tended to have recurrence within 1 year. Patients who had recurrence more than
\end{abstract}

I. Araki - K. Hosoda - K. Yamashita - N. Katada - H. Moriya

H. Mieno · A. Ema · S. Kikuchi · M. Watanabe $(\square)$

Department of Surgery, School of Medicine, Kitasato University,

Kitasato1-15-1, Minami-ku, Sagamihara, Kanagawa 252-0374,

Japan

e-mail: gekaw@med.kitasato-u.ac.jp

S. Sakuramoto

Department of Gastrointestinal Surgery, Saitama Medical

University International Medical Center, Yamane 1397-1,

Hidaka, Saitama 350-1298, Japan

T. Mikami

Department of Pathology, Toho University School of Medicine,

Omori-Nishi 5-21-16, Ohta, Tokyo 143-8540, Japan
2 years after surgery tended to survive for longer than 5 years after recurrence. Moderate or marked venous invasion (v2 or v3) and age $>65$ years were significantly associated with relapse-free and overall survival on univariate analysis. On multivariate analysis, the only independent prognostic factor for relapse-free and overall survival was venous invasion.

Conclusions Moderate or marked venous invasion (v2 or v3) is an independent predictor of relapse-free and overall survival in stage IB node-negative gastric cancer. Postoperative adjuvant chemotherapy, currently not given to this subgroup of patients, may improve the outcomes of patients with stage IB node-negative gastric cancer, particularly when accompanied by venous invasion.

Keywords Gastric cancer - Stage IB - Prognostic factors · Venous invasion $\cdot$ Pattern of recurrence

\section{Introduction}

Gastric cancer is the fourth most common malignancy (989,000 cases in 2008) and the second leading cause of cancer-related death (737,000 deaths) worldwide [1], with the highest incidence rates are in East Asia, East Europe, and South America [2]. In Japan, the incidence rate of gastric cancer remains relatively high, although mortality from gastric cancer has decreased significantly as the result of progress in diagnosis and therapy, including improvements in gastric cancer screening, surgery, and chemotherapy. In stage IA gastric cancer as defined by the Japanese Classification of Gastric Carcinoma [3], the 5-year survival rate is reported to be $92 \%$ [4], suggesting no need for adjuvant therapy. In stage II or III advanced gastric cancer, D2 gastrectomy and adjuvant chemotherapy 
with S1 have prolonged overall survival and relapse-free survival $[5,6]$. Although standard therapy for stage IB advanced gastric cancer is surgery alone in Japan, a certain proportion of patients have recurrence, and nearly all patients with recurrence die of their disease. If we could identify patients with stage IB gastric cancer who are at high risk for recurrence and death, such patients might benefit from adjuvant chemotherapy.

Undifferentiated-type adenocarcinoma has been reported to be the only risk factor for recurrence in patients with stage IB gastric cancer [7]. However, this finding was based on studies including patients who had T1 (early) gastric cancer with regional lymph node metastasis, and little is known about the risk factors for recurrence in stage IB advanced (T2 or T3) gastric cancer without lymph node metastasis. We conducted this retrospective study to identify clinicopathological prognostic factors and to clarify recurrence patterns in patients with stage IB nodenegative gastric cancer.

\section{Materials and methods}

\section{Patients}

We retrospectively reviewed the medical records of 1532 patients with primary gastric cancer who underwent gastrectomy at Kitasato University East Hospital between January 2000 and December 2010. All patients had undergone gastrectomy with sufficient lymph-node dissection, and 142 were histopathologically confirmed to have stage IB gastric cancer without lymph node metastasis on routine hematoxylin and eosin staining (pT2pN0), as defined in the 13th edition of the Japanese Classification of Gastric Carcinoma [3]. One patient was lost to follow-up because of no return visit to the hospital, 1 underwent esophagectomy in addition to gastrectomy because of advanced esophageal cancer in addition to gastric cancer, and 10 had received neoadjuvant chemotherapy. These 12 patients were excluded from the study, and the remaining 130 patients were included in the analysis. This study was conducted in accordance with the Declaration of Helsinki, and all patients signed a consent form approved by the Research Ethics Committee of Kitasato University School of Medicine.

\section{Pathological diagnosis}

Histopathologically, papillary and tubular adenocarcinomas were grouped together as differentiated-type adenocarcinoma, and poorly differentiated and mucinous adenocarcinomas and signet-ring cell carcinomas were classified as undifferentiated-type adenocarcinoma. The degrees of lymphatic invasion (from ly0 to ly 3 ) and venous invasion (from v0 to v3) were defined according to the Japanese Classification of Gastric Carcinoma [3]. Venous invasion was evaluated on histopathological evaluation of specimens stained with hematoxylin and eosin and with elastica van Gieson stain (Fig. 1). Lymphatic invasion was evaluated on histopathological evaluation of specimens stained with hematoxylin and eosin. In our hospital, v0, v1, $\mathrm{v} 2$, and $\mathrm{v} 3$ are defined as follows: v0, no venous invasion found on any slide examined; v1, one or two sites of venous invasion found throughout all slides examined (eight slides); v2, intermediate level between v1 and v3; $\mathrm{v} 3$, one or more sites of venous invasions found on every slide examined. Ly0, ly1, ly2, and ly3 are defined in the same way.

Immunohistochemical staining for HER2 in nodenegative gastric cancer with recurrence

We conducted immunohistochemistry staining for HER2 in node-negative stage IB gastric cancer patients with recurrence. Staining was evaluated using light microscopy and scored using a $4-$ graded scale $(0,1+, 2+$, or $3+)$. We followed the consensus panel recommendations for HER2 scoring in gastric cancer [8].

\section{Patient follow-up}

After gastrectomy, the patients were generally assessed at 6-month intervals for the first 5 years and at 12-month intervals thereafter. The baseline assessment included physical examination, hematological examination, serum chemical analysis, measurement of carcinoembryonic antigen (CEA) levels, computed tomography (CT) of the chest and abdomen, and gastroscopic examination. Recurrence was diagnosed on the basis of imaging studies and cytological analysis. The median follow-up was 59 months. The clinicopathological characteristics of the 130 patients are shown in Table 1.

Statistical analysis

Fisher's exact test was used to analyze categorical variables. Survival was calculated by the Kaplan-Meier method. Univariate analyses of prognostic factors associated with relapse-free and overall survival were performed using logrank tests. Factors with significant effects on relapse-free and overall survival on the univariate analyses were then subjected to multivariate analysis using a Cox proportional hazards model to identify independent prognostic factors. All calculations were performed with the use of JMP 10 software (SAS Institute, Cary, NC, USA). $P$ values $<0.05$ were considered to indicate statistical significance. 

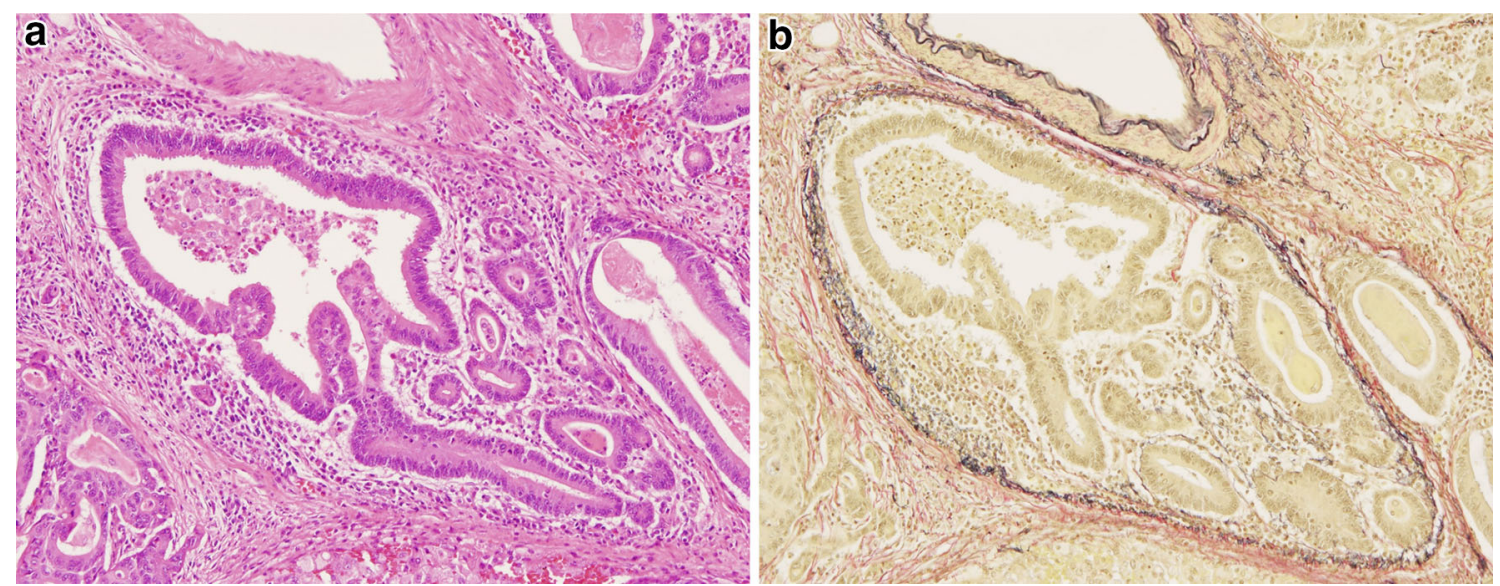

Fig. 1 a Hematoxylin and eosin stain. b Elastica van Gieson stain. Venous invasion is easily identified because the elastic fibers of veins are stained dark violet with elastica van Gieson stain

\section{Results}

Recurrence rate and mortality

Of the 130 patients who underwent surgery for stage IB node-negative gastric cancer, $12(9.2 \%)$ had recurrence. The clinicopathological characteristics of the patients with recurrence are shown in Table 2 . Ten $(83 \%)$ of the 12 patients with recurrence died. Four patients $(3.1 \%)$ without recurrence died of other diseases (lung cancer, 1; bladder cancer, 1; pneumonia, 1; unknown, 1). The rates of relapse-free survival and overall survival at 5 years were $88 \%$ and $89 \%$, respectively. One of the patients who survived after recurrence had liver metastasis. He received radiofrequency ablation therapy and had a complete response. Another survivor received chemotherapy with S1 for 6 months after the detection of metastasis. Subsequent cancer therapy was precluded by severe adverse events, such as nausea and vomiting. However, her condition remained stable, with no further treatment.

Of the 12 recurrent patients, only $1(8 \%)$ patient had a tumor with high level of HER2 protein (immunohistochemistry $3+$ ), whereas the remaining 11 (92\%) patients had tumors with negative HER2 status (immunohistochemistry 0).

Initial sites of recurrence and timing of recurrence

The initial sites and the timing of recurrence are shown in Table 3. Of the 12 patients with recurrence, 7 (58\%) had liver metastasis, $3(25 \%)$ had distant lymph node metastasis, $2(17 \%)$ had peritoneal dissemination, and $1(8.3 \%)$ had locoregional recurrence. One patient had recurrence in the liver and distant lymph nodes simultaneously. Of the 7 patients who had liver metastasis, 3 had differentiated-type adenocarcinoma and 4 had undifferentiated-type adenocarcinoma. There was no association between liver metastasis and histological differentiation $(P=0.58)$. Moreover, moderate or marked venous invasion (v2 or v3) was not associated with liver metastasis $(P=0.58)$.

The timing of recurrence was characterized by a biphasic pattern (Fig. 2a). Early recurrence occurred within 2 years after surgery, whereas late recurrence occurred between 4 and 5 years after surgery. The initial sites of recurrence did not differ between early recurrence and late recurrence (Table 3). Of the six patients who had recurrence within 1 year after surgery, $4(67 \%)$ had tumors more than $5 \mathrm{~cm}$ in diameter. In contrast, of the six patients who had recurrence more than 1 year after surgery, only one $(1.7 \%)$ had a tumor more than $5 \mathrm{~cm}$ in diameter (Fig. $2 b, P=0.24$ ). All three patients who survived for more than 5 years after recurrence had recurrence more than 2 years after surgery. Among the seven patients who died within 5 years after recurrence, only one $(14 \%)$ had recurrence more than 2 years after surgery (Fig. 2c, $P=0.033$ ).

Clinicopathological variables associated with overall and relapse-free survival

\section{Univariate analysis}

The results of the univariate analyses for factors associated with overall survival and relapse-free survival in stage IB node-negative gastric cancer are summarized in Table 4 and Table 5, respectively. Moderate or marked venous invasion (v2 or v3) and age >65 years were significantly associated with overall and relapse-free survival. Moreover, gender tended to be associated with overall survival. Tumor location, tumor size, histology, 
Table 1 Clinicopathological characteristics $(n=130)$

\begin{tabular}{|c|c|}
\hline \multicolumn{2}{|l|}{ Age (years) } \\
\hline Mean $\pm \mathrm{SD}$ & $65.5 \pm 11.9$ \\
\hline Median (range) & $66.5(21-90)$ \\
\hline \multicolumn{2}{|l|}{ Gender } \\
\hline Male & 98 \\
\hline Female & 32 \\
\hline \multicolumn{2}{|l|}{ Location } \\
\hline Upper & 36 \\
\hline Middle & 59 \\
\hline Lower & 35 \\
\hline \multicolumn{2}{|l|}{ Tumor size $(\mathrm{cm})$} \\
\hline Mean $\pm \mathrm{SD}$ & $5.0 \pm 2.7$ \\
\hline Median (range) & $4.5(1.0-16)$ \\
\hline \multicolumn{2}{|l|}{ Histology } \\
\hline Differentiated & 61 \\
\hline Undifferentiated & 69 \\
\hline \multicolumn{2}{|l|}{ Depth of tumor invasion } \\
\hline $\mathrm{mp}$ & 66 \\
\hline ss & 64 \\
\hline \multicolumn{2}{|l|}{ Lymphatic invasion (ly) } \\
\hline 0 & 33 \\
\hline 1 & 76 \\
\hline 2 & 20 \\
\hline 3 & 1 \\
\hline \multicolumn{2}{|l|}{ Venous invasion (v) } \\
\hline 0 & 30 \\
\hline 1 & 47 \\
\hline 2 & 35 \\
\hline 3 & 18 \\
\hline \multicolumn{2}{|l|}{ Adjuvant chemotherapy } \\
\hline Yes & 6 \\
\hline No & 124 \\
\hline Patients with recurrence & $12(9.2 \%)$ \\
\hline Five-year relapse-free survival rate & $88 \%$ \\
\hline Five-year overall survival rate & $89 \%$ \\
\hline
\end{tabular}

$m p$ muscularis propria, ss subserosal

depth of tumor invasion, lymphatic invasion, and adjuvant chemotherapy were not associated with overall or relapse-free survival.

\section{Multivariate analysis}

To determine independent prognostic factors associated with overall and relapse-free survival in patients with stage IB node-negative gastric cancer, probable factors on univariate analysis were input into a Cox proportiona hazards model (Tables 4, 5). The only independent prognostic factor for overall survival and relapse-free survival was moderate or marked venous invasion $(P=0.006$, HR 5.00,

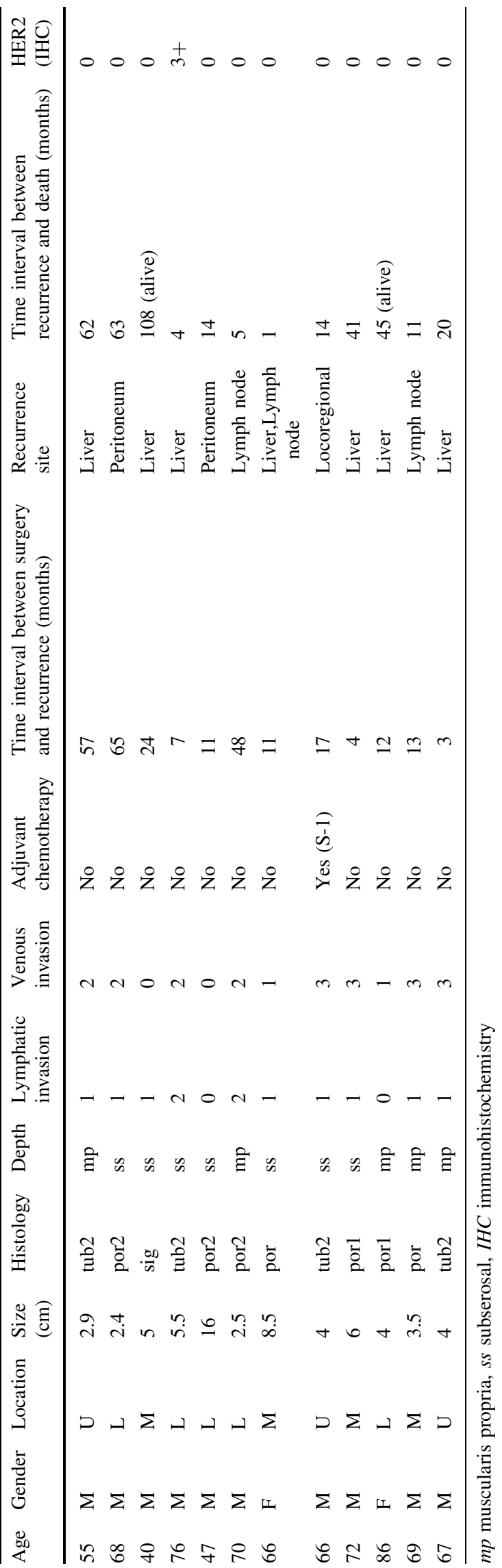


Table 3 First site and the timing of recurrence $(n=12)$

\begin{tabular}{lllll}
\hline & Early & Late & Total & $\%$ \\
\hline Liver & 5 & 1 & 6 & 50 \\
Distant lymph nodes & 1 & 1 & 2 & 17 \\
Peritoneum & 1 & 1 & 2 & 17 \\
Locoregional & 1 & 0 & 1 & 8.3 \\
Mixed type & 1 & 0 & 1 & 8.3 \\
\hline
\end{tabular}

Mixed type liver metastasis and distant lymph node metastasis

Early within 2 years after surgery

Late between 4 years and 5 years after surgery

$95 \%$ CI $1.54-22.36 ; \quad P=0.035, \quad$ HR $3.00,95 \%$ CI $1.08-9.59$, respectively).

\section{Survival curves}

Kaplan-Meier curves for overall survival and relapse-free survival are shown in Fig. 3a, b, respectively. The 5-year overall survival rate of patients with moderate or marked venous invasion ( $83 \%$ ) was significantly lower than that of patients with no or minimal venous invasion $(96 \%$; logrank test, $P=0.002$ ). In addition, the 5-year relapse-free survival rate of patients with moderate or marked venous invasion $(80 \%)$ was significantly lower than that of the patients with no or minimal venous invasion $(93 \%$; $\log$ rank test, $P=0.014)$.

\section{Discussion}

In our retrospective study, the only independent prognostic factor associated with overall and relapse-free survival was moderate or marked venous invasion (v2 or v3) in patients with stage IB node-negative gastric cancer. Several previous studies have identified a number of prognostic factors for all stages of gastric cancer, and lymph node metastasis was reported to be the strongest prognostic factor in gastric adenocarcinoma after $\mathrm{R} 0$ resection [9]. Although nodenegative gastric cancer has better outcomes than nodepositive disease [10], recurrence and metastasis still occur in a subset of patients after curative resection. For example, Kunisaki et al. [11] reported that age ( $\geq 60$ years), poorly defined macroscopic appearance, and undifferentiated histological type were independently related to lower survival rates in patients with pN0 gastric cancer. Baiocchi et al. [12] found that infiltration depth and histological type were

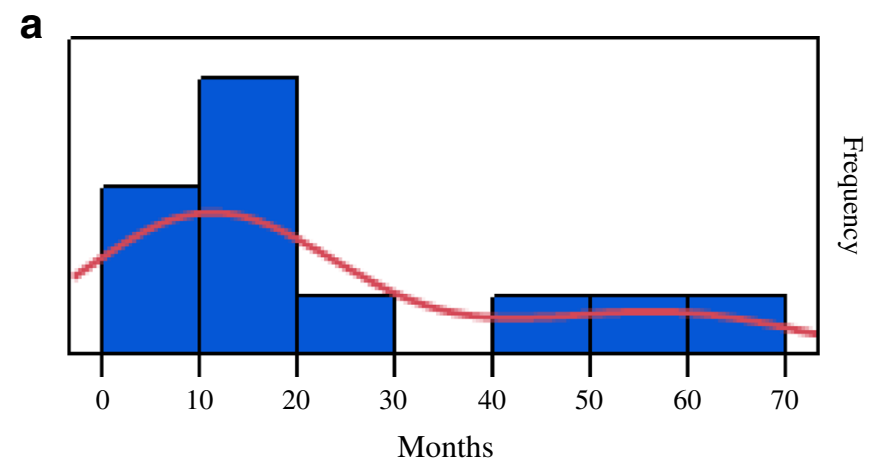

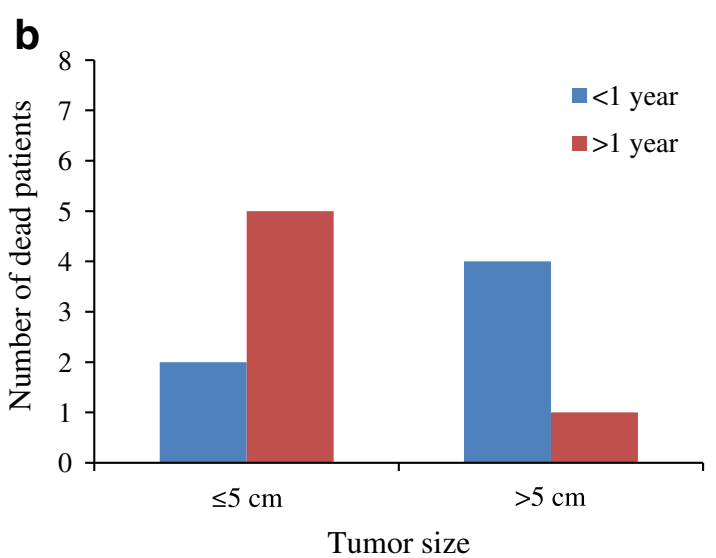

Fig. 2 a Biphasic pattern of recurrence in stage IB node-negative gastric cancer. b Number of patients who had recurrence less than 1 year or more than 1 year after surgery according to tumor size

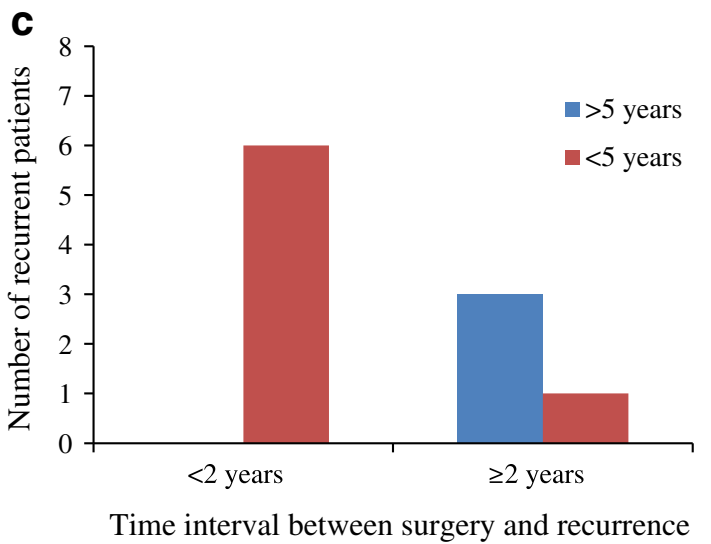

$(P=0.24)$. c Number of patients who died more than 5 years or $<5$ years after recurrence according to the time interval between surgery and recurrence $(P=0.033)$ 
Table 4 Univariate and prognostic factors for overall survival multivariate analyses of
* Indicates statistical significance; $P<0.10$

$O S$ overall survival, $H R$ hazard ratio, $C I$ confidence interval

\begin{tabular}{|c|c|c|c|c|c|c|}
\hline \multirow[t]{2}{*}{ Variable } & \multicolumn{3}{|c|}{ Univariate analysis } & \multicolumn{3}{|c|}{ Multivariate analysis } \\
\hline & $n$ & 5-year OS (\%) & $P$ value & $\mathrm{HR}$ & $95 \% \mathrm{CI}$ & $P$ value \\
\hline Age (years) & & & $0.027 *$ & & & 0.080 \\
\hline$>65$ & 72 & 85 & & 2.92 & $0.89-13.09$ & \\
\hline$\leq 65$ & 58 & 96 & & 1.00 & & \\
\hline Gender & & & 0.13 & & & \\
\hline Male & 98 & 90 & & & & \\
\hline Female & 32 & 97 & & & & \\
\hline Location & & & 0.70 & & & \\
\hline Upper & 35 & 89 & & & & \\
\hline Middle & 59 & 91 & & & & \\
\hline Lower & 36 & 90 & & & & \\
\hline Tumor size & & & 0.93 & & & \\
\hline$>5 \mathrm{~cm}$ & 56 & 88 & & & & \\
\hline$\leq 5 \mathrm{~cm}$ & 74 & 93 & & & & \\
\hline Histology & & & 0.33 & & & \\
\hline Differentiated & 61 & 89 & & & & \\
\hline Undifferentiated & 69 & 92 & & & & \\
\hline Depth & & & 0.26 & & & \\
\hline $\mathrm{mp}$ & 66 & 93 & & & & \\
\hline ss & 64 & 88 & & & & \\
\hline Lymphatic invasion & & & 0.65 & & & \\
\hline ly0 or ly 1 & 109 & 91 & & & & \\
\hline ly 2 or $1 y 3$ & 21 & 89 & & & & \\
\hline Venous invasion & & & $0.002 *$ & & & $0.006^{*}$ \\
\hline $\mathrm{v} 0$ or $\mathrm{v} 1$ & 77 & 96 & & 1.00 & & \\
\hline v 2 or v3 & 53 & 83 & & 5.00 & $1.54-22.36$ & \\
\hline Adjuvant chemotherapy & & & 0.49 & & & \\
\hline Yes & 6 & 80 & & & & \\
\hline No & 124 & 91 & & & & \\
\hline
\end{tabular}

the only independent predictors of survival in patients with node-negative gastric cancer. However, these results included patients with serosal invasion, and a large proportion of patients with recurrence had peritoneal dissemination.

In our study, 12 patients had recurrence. Seven of these patients had recurrence in the liver caused by hematogenous metastasis. An association between hematogenous metastasis and differentiated-type adenocarcinoma has been reported [11, 13], but was not evident in our study, perhaps because our study group was too small to reach statistical significance. Recurrence was associated with distant lymph node metastasis in three patients. These patients had lymphatic invasion of ly1 or more. The lymphatic systems of these patients were thus involved by carcinoma cells, and micrometastasis in harvested lymph nodes might have been overlooked on routine histological examination. Micrometastases to regional lymph nodes have been reported to affect the outcomes of patients with pN0 gastric cancer [14]. However, some studies have suggested that there is no relationship between micrometastasis and outcomes $[15,16]$. Nonetheless, it is important to take micrometastasis into account when following up such patients.

Of the 12 recurrent patients, only $1(8 \%)$ patient had a tumor with HER2 positivity (immunohistochemistry $3+$ ). This figure of $8 \%$ fell within the previously reported range of 4-53\% [17]. Some studies have suggested that HER2 status in gastric cancer is associated with poor outcomes and aggressive disease $[18,19]$. However, HER2 positivity did not necessarily seem to be associated with poor survival in our study.

Our study showed that stage IB node-negative gastric cancer was associated with a biphasic pattern of recurrence. A time-varying pattern of recurrence risk has been reported previously for all stages of gastric cancer [20]. In that study, the initial major recurrence peak covered the first 3 years after surgery, followed by a decline until 7.5 years after the surgery, after which the curve began to rise again. This recurrence pattern was speculated to be 
Table 5 Univariate and multivariate analyses of prognostic factors for relapsefree survival
* Indicates statistical significance; $P<0.10$ $R F S$ relapse-free survival, $H R$ hazard ratio, $C I$ confidence interval

\begin{tabular}{|c|c|c|c|c|c|c|}
\hline \multirow[t]{2}{*}{ Variable } & \multicolumn{3}{|c|}{ Univariate analysis } & \multicolumn{3}{|c|}{ Multivariate analysis } \\
\hline & $n$ & 5-year RFS (\%) & $P$ value & HR & $95 \% \mathrm{CI}$ & $P$ value \\
\hline Age (years) & & & $0.044^{*}$ & & & 0.088 \\
\hline$>65$ & 72 & 84 & & 2.55 & $0.88-9.19$ & \\
\hline$\leq 65$ & 58 & 92 & & 1.00 & & \\
\hline Gender & & & 0.26 & & & \\
\hline Male & 98 & 86 & & & & \\
\hline Female & 32 & 94 & & & & \\
\hline Location & & & 0.83 & & & \\
\hline Upper & 35 & 85 & & & & \\
\hline Middle & 59 & 90 & & & & \\
\hline Lower & 36 & 86 & & & & \\
\hline Tumor size & & & 0.87 & & & \\
\hline$>5 \mathrm{~cm}$ & 56 & 87 & & & & \\
\hline$\leq 5 \mathrm{~cm}$ & 74 & 88 & & & & \\
\hline Histology & & & 0.87 & & & \\
\hline Differentiated & 61 & 87 & & & & \\
\hline Undifferentiated & 69 & 88 & & & & \\
\hline Depth & & & 0.24 & & & \\
\hline $\mathrm{mp}$ & 66 & 88 & & & & \\
\hline ss & 64 & 87 & & & & \\
\hline Lymphatic invasion & & & 0.56 & & & \\
\hline ly0 or ly1 & 109 & 87 & & & & \\
\hline ly 2 or ly 3 & 21 & 90 & & & & \\
\hline Venous invasion & & & $0.014^{*}$ & & & $0.035^{*}$ \\
\hline $\mathrm{v} 0$ or v1 & 77 & 93 & & 1.00 & & \\
\hline v2 or v3 & 53 & 80 & & 3.00 & $1.08-9.59$ & \\
\hline Adjuvant chemotherapy & & & 0.72 & & & \\
\hline Yes & 6 & 83 & & & & \\
\hline No & 124 & 88 & & & & \\
\hline
\end{tabular}
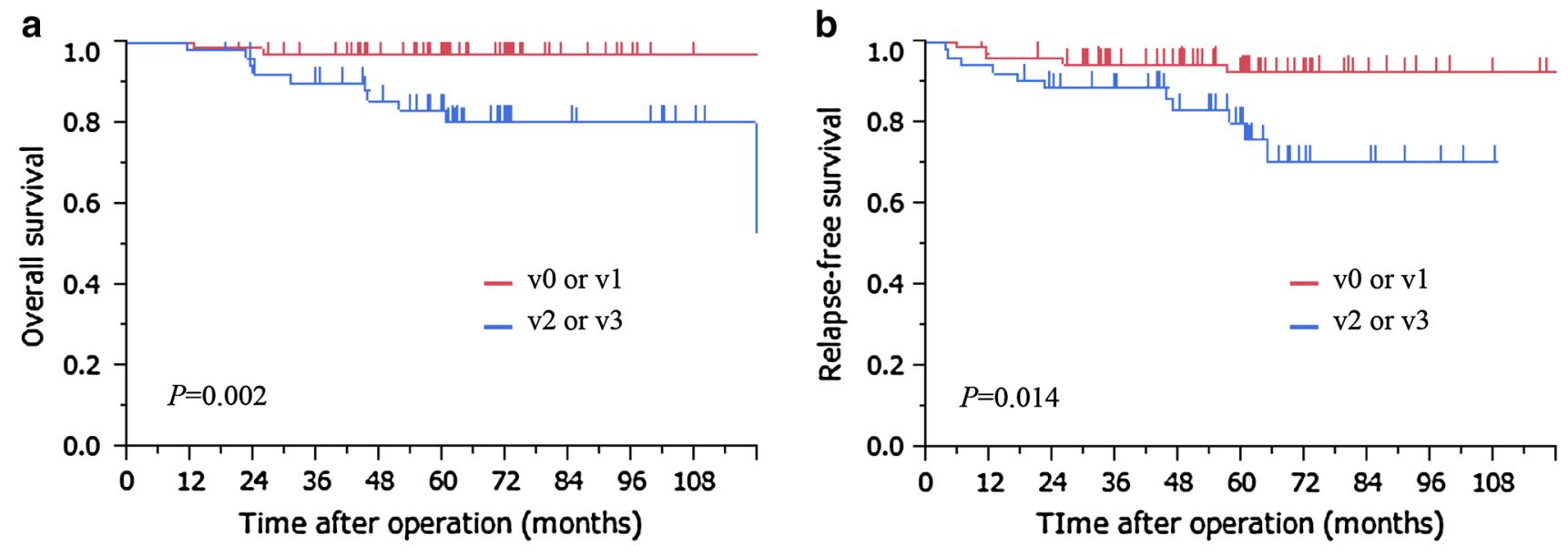

Fig. 3 a Overall survival of patients with stage IB gastric cancer according to the degree of venous invasion (log-rank test, $P=0.002)$. b Relapse-free survival of patients with stage IB gastric cancer according to the degree of venous invasion $($ log-rank test, $P=0.014)$ 
caused by tumor dormancy. Although the exact reasons underlying this pattern remain unknown, we consider it important to closely follow patients with gastric cancer for more than 5 years.

Our results also demonstrated that patients with tumors more than $5 \mathrm{~cm}$ in diameter tended to have recurrence within 1 year. One such patient with no lymphatic or venous invasion had peritoneal recurrence. The primary tumor was scirrhous type, and patients with this type of tumor should be closely observed for peritoneal recurrence. In any case, it is important to carefully assess such patients within 1 year after surgery according to the status of lymphatic and venous invasion. On the other hand, patients with primary tumors $<5 \mathrm{~cm}$ in diameter tended to have recurrence more than 1 year after surgery. It is important to follow up these patients closely, especially from 1 year after surgery.

An interesting finding of our study was that patients who had recurrence more than 2 years after surgery had a significantly higher probability of surviving for more than 5 years after recurrence than those who had recurrence less than 2 years after surgery. This finding suggests that patients who have recurrence more than 2 years after surgery should receive multidisciplinary therapy.

In patients with gastric cancer, complete resection of the primary tumor, including an adequate resection margin and lymph node dissection to reduce the risk of locoregional recurrence, is considered standard treatment. However, the relatively high rate of recurrence in patients with moderate or marked venous invasion (v2 or v3) in our study suggests that surgery alone is inadequate, and other treatment approaches are needed to reduce recurrence. Eleven of the 12 patients with recurrence did not receive adjuvant chemotherapy. If patients with moderate or marked venous invasion (v2 or v3) had received adjuvant chemotherapy, their overall survival might have been improved.

According to the ACTS-GC study, S-1 monotherapy for 1 year has become standard adjuvant chemotherapy after curative D2 gastrectomy in Japan. However, subset analysis revealed that S-1 monotherapy could hardly have controlled hematogenous metastasis with metastatic rate of $11.5 \%(61 / 529)$ in the S-1 group as compared with that of $13.4 \%(71 / 530)$ in the surgery-only group. On the other hand, combined chemotherapy using capecitabine and oxaliplatin have prolonged disease-free survival after curative D2 gastrectomy in the CLASSIC study [21], where subset analysis revealed that this combination chemotherapy could well have controlled distant site metastasis with metastatic rate of $9.4 \%(49 / 520)$ in the chemotherapy group as compared with $15.1 \%(78 / 515)$ in the surgeryonly group. In our study, 7 of 12 recurrent patients had hematogenous metastasis, so adjuvant chemotherapy using capecitabine and oxaliplatin might be appropriate for the patients with high-risk stage IB node-negative gastric cancer patients.

In our study, the only independent prognostic factor for stage IB node-negative gastric cancer was moderate or marked venous invasion, which could only be obtained after surgical resection. If some factors obtained preoperatively were identified to be indicative for poor prognosis, patients with such factors could be recommended to receive neoadjuvant chemotherapy in the future.

In our institute, elastica van Gieson stain is routinely performed in pathological examination for all gastrointestinal surgery patients to identify venous invasion. In light of our results, we think that elastica van Gieson stain is necessary for accurate diagnosis of venous invasion. On the other hand, D2-40 staining is not routinely performed in our institute to identify lymphatic invasion. There are some cases for whom D2-40 staining is important (i.e., patients with gastric cancer of submucosal invasion who underwent endoscopic submucosal dissection). However, our study could not have identified lymphatic invasion as an independent prognostic factor.

Our study had several important limitations. First, it was a small, retrospective, case-control study performed at a single institution. Second, follow-up strategies, including the timing of diagnostic imaging studies and the use of adjuvant chemotherapy, differed slightly among the patients' outpatient physicians.

In conclusion, our study demonstrated that moderate or marked venous invasion ( $\mathrm{v} 2$ or $\mathrm{v} 3$ ) was an independent predictor of relapse-free and overall survival in patients with stage IB node-negative gastric cancer. Postoperative adjuvant chemotherapy may be a reasonable approach to improving the outcomes of such patients, particularly in the presence of venous invasion. Further prospective clinical trials are needed to confirm our results.

\section{References}

1. International Agency for Research on Cancer; GLOBOCAN2008.

2. Jemal A, Bray F, Center MM, Ferlay J, Ward E, Forman D. Global cancer statistics. CA Cancer J Clin. 2011;61:69-90.

3. Japanese Gastric Cancer Association. Japanese classification of gastric carcinoma, 2nd English edition. Gastric Cancer. 1998;1:10-24.

4. Nashimoto A, Akazawa K, Isobe Y, Miyashiro I, Katai H, Kodera Y, Tsujitani S, Seto Y, Furukawa H, Oda I, Ono H, Tanabe S, Kaminishi M. Gastric cancer treated in 2002 in Japan: 2009 annual report of the JGCA nationwide registry. Gastric Cancer. 2013;16:1-27.

5. Sakuramoto S, Sasako M, Yamaguchi T, Kinoshita T, Fujii M, Nashimoto A, Furukawa H, Nakajima T, Ohashi Y, Imamura H, Higashino M, Yamamura Y, Kurita A, Arai K, ACTS-GC Group. Adjuvant chemotherapy for gastric cancer with S-1, an oral fluoropyrimidine. N Engl J Med. 2007;357:1810-20. 
6. Sasako M, Sakuramoto S, Katai H, Kinoshita T, Furukawa H, Yamaguchi T, Nashimoto A, Fujii M, Nakajima T, Ohashi Y. Five-year outcomes of a randomized phase III trial comparing adjuvant chemotherapy with S-1 versus surgery alone in stage II or III gastric cancer. J Clin Oncol. 2011;29:4387-93.

7. Yokoyama T, Kamada K, Tsurui Y, Kashizuka H, Okano E, Ogawa S, Obara S, Tatsumi M. Clinicopathological analysis for recurrence of stage $\mathrm{Ib}$ gastric cancer (according to the second English edition of the Japanese classification of gastric carcinoma). Gastric Cancer. 2011;14:372-7.

8. Hofmann M, Stoss O, Shi D, Buttner R, van de Vijver M, Kim W, Ochiai A, Ruschoff J, Henkel T. Assessment of a HER2 scoring system for gastric cancer: results from a validation study. Histopathology (Oxf). 2008;52:797-805.

9. Hartgrink HH, Jansen EP, van Grieken NC, van de Velde CJ. Gastric cancer. Lancet. 2009;374:477-90.

10. Dicken BJ, Bigam DL, Cass C, Mackey JR, Joy AA, Hamilton SM. Gastric adenocarcinoma: review and considerations for future directions. Ann Surg. 2005;241:27-39.

11. Kunisaki C, Shimada H, Nomura M, Matsuda G, Otsuka Y, Ono $\mathrm{H}$, Akiyama $\mathrm{H}$. Therapeutic strategy for patients with $\mathrm{pN} 0$ gastric carcinoma. J Surg Oncol. 2006;94:212-9.

12. Baiocchi GL, Tiberio GA, Minicozzi AM, Morgagni P, Marrelli D, Bruno L, Rosa F, Marchet A, Coniglio A, Saragoni L, Veltri M, Pacelli F, Roviello F, Nitti D, Giulini SM, De Manzoni G. A multicentric Western analysis of prognostic factors in advanced, node-negative gastric cancer patients. Ann Surg. 2010;252:70-3.

13. Kunisaki C, Shimada H, Nomura M, Matsuda G, Otsuka Y, Ono $\mathrm{H}$, Akiyama H. Surgical outcome of serosa-negative advanced gastric carcinoma. Anticancer Res. 2004;24:3169-75.

14. Nakajo A, Natsugoe S, Ishigami S, Matsumoto M, Nakashima S, Hokita S, Baba M, Takao S, Aikou T. Detection and prediction of micrometastasis in the lymph nodes of patients with pN0 gastric cancer. Ann Surg Oncol. 2001;8:158-62.

15. Morgagni P, Saragoni L, Folli S, Gaudio M, Scarpi E, Bazzocchi F, Marra GA, Vio A. Lymph node micrometastases in patients with early gastric cancer: experience with 139 patients. Ann Surg Oncol. 2001;8:170-4.

16. Bozzetti F, Andreola S, Bignami P, Sirizzotti G. Prognostic effects of lymph node micrometastases in patients undergoing curative gastrectomy for cancer. Tumori. 2000;86:470-1.

17. Chua TC, Merrett ND. Clinicopathologic factors associated with HER2-positive gastric cancer and its impact on survival outcomes-a systematic review. Int J Cancer. 2012;130:2845-56.

18. Gravalos C, Jimeno A. HER2 in gastric cancer: a new prognostic factor and a novel therapeutic target. Ann Oncol. 2008;19: 1523-9.

19. Tanner M, Hollmen M, Junttila TT, Kapanen AI, Tommola S, Soini Y, Helin H, Salo J, Joensuu H, Sihvo E, Elenius K, Isola J. Amplification of HER-2 in gastric carcinoma: association with topoisomerase II-alpha gene amplification, intestinal type, poor prognosis and sensitivity to trastuzumab. Ann Oncol. 2005;16: $273-8$.

20. Feng XY, Chen YB, Wang W, Guan YX, Li YF, Li YF, Chen S, Sun XW, Li W, Xu DZ, Han YQ, Zhang XS, Zhou ZW. Timevarying pattern of recurrence risk for gastric cancer patients. Med Oncol. 2013;30:514

21. Bang YJ, Kim YW, Yang HK, Chung HC, Park YK, Lee KH, Lee KW, Kim YH, Noh SI, Cho JY, Mok YJ, Kim YH, Ji J, Yeh TS, Button P, Sirzen F, Noh SH, CLASSIC trial investigators. Adjuvant capecitabine and oxaliplatin for gastric cancer after D2 gastrectomy (CLASSIC): a phase 3 open-label, randomised controlled trial. Lancet. 2012;379:315-21. 